\title{
FINITE ELEMENT METHOD FOR THE ACCURATE SOLUTION OF DIATOMIC
} MOLECULES

\author{
D. Heinemann, B. Fricke, D. Kolb \\ Physics Department \\ University of Kassel \\ Heinrich Plett Str. 40 \\ D-3500 Kasse 1 \\ Germany
}

ABSTRACT. We present the Finite-Element-Method (FEM) in its application to quantum mechanical problems solving for diatomic molecules. Results for Hartree-Fock calculations of $\mathrm{H}_{2}$ and Hartree-Fock-Slater calculations of molecules like $\mathrm{N}_{2}$ and $\mathrm{CO}$ have been obtained. The accuracy achieved with less then 5000 grid points for the total energies of these systems is $10^{-8}$ a.u. , which is demonstrated for $\mathrm{N}_{2}$.

\section{INTRODUCTION}

The Finite Element Method (FEM) is a well known technique for solving static and dynamical problems in engineering science. Examples can be found in the textbooks of Ref. 13. The idea of this method is to divide the space into a number of 2 or 3 dimensional domains and describe the properties of interest on each of these elements separately connected via boundary conditions. In engineering science one uses low order polynomials to specify the interesting quantities on each element.

We applied the FEM to quantum mechanical problems where the accuracy required is very high. To achieve this accuracy we were forced to use high order polynomials on each element and, in order to minimize the total number of points, to use a small number of elements.

\section{THE HARTREE-FOCK-SLATER EQUATIONS}

The Hartree-Fock equations for a diatomic molecule read:

$$
H_{H F}(\underline{r}) \varphi_{i}(\underline{r})=\varepsilon_{i} \varphi_{i}(\underline{r}) \quad ; i=1,2, \ldots, N \text {. }
$$


With

$$
\begin{aligned}
& H_{H F}(\underline{r})=h(\underline{r})+V^{C}(\underline{r})+V^{X}(\underline{r}) \\
& h(\underline{r})=-\nabla^{1} \nabla^{2}-\frac{Z_{1}}{\mid} \underline{r}_{1}-\underline{r}|-| \underline{r}_{1}-\underline{r} \mid \\
& V^{C}(\underline{r})=\sum_{i=1}^{N} \int_{j}^{*}\left(\underline{r}^{\prime}\right)-\underline{r}-\underline{r}^{\prime} \varphi_{j}\left(\underline{r}^{\prime}\right) d \underline{r}^{\prime}
\end{aligned}
$$

and

$$
V^{x}(\underline{r}) \quad \varphi_{j}(\underline{r})=\sum_{i=1}^{N} \int \varphi_{i}^{*}\left(\underline{r}^{\prime}\right)-\underline{1}-\underline{r}^{\prime} \mid \rho_{j}\left(\underline{r}^{\prime}\right) d \underline{r}^{\prime} \varphi_{j}(\underline{r}) .
$$

The interelectronic coulomb potential $V^{C}(r)$ satisfies the Poisson equation

$$
\nabla^{2} V^{c}(\underline{r})=-4 \pi \rho(\underline{r})
$$

where $\rho(\underline{r})$ is the total electronic density.

In the Hartree-Fock-Slater equations the nonlocal exchange potential $V^{x}(\underline{r})$ of the Hartree-Fock equations is replaced by the local exchange potential

$$
v^{x}(\underline{r})=-3 / 2 \alpha(3 / \pi \rho(\underline{r}))^{1 / 3}
$$

where $\alpha$ is a constant. We use this simplified form of a local exchange with $\alpha=0.7$.

The total molecular energy then is

$$
\begin{aligned}
E_{\text {tot }}=\sum_{i=1}^{N} \varepsilon_{i} & -1 / 2 \int \rho(\underline{r}) v^{c}(\underline{r}) d \underline{r}- \\
& -1 / 4 \int \rho(\underline{r}) v^{x}(\underline{r}) d \underline{r}
\end{aligned}
$$

The differential equations to be solved are the Schrödinger type equation derived from Equ. (1) with potentials kept fixed, and the poisson equation (4) relating the potential $v^{c}$ to the electronic density

\section{THE COORDINATE SYSTEM}

To solve the Hartree-Fock-Slater differential equations for a diatomic molecule numerically we chose the prolate spheroidal coordinates 


$$
\begin{aligned}
& x_{1}=R / 2 \sinh (s) \sin (t) \cos (0) \\
& x_{2}=R / 2 \sinh (s) \sin (t) \sin (p) \\
& x_{3}=R / 2 \cosh (s) \cos (t)
\end{aligned}
$$

which where also used by Laaksonen et al. ${ }^{4}$ and Becke ${ }^{5}$ for their num erical approach. Within these coordinates the unknown one electron wave functions are:

$$
\varphi_{j}(s, t,)=\varphi_{j}(s, t) \exp (i m \omega) ;
$$

$m$ denotes the projection of the angular momentum onto the internuclear axis.

Using this ansatz the Schrödinger type equation (1) can be rewritten as a two dimensional differential equation

$$
\begin{aligned}
& -\frac{1}{2}-\frac{\partial}{\partial s}\left(K_{1}(s, t) \frac{\partial \varphi_{j}}{\partial s}\right)-\frac{1}{\partial s} \frac{\partial}{2} \frac{-}{\partial t}\left(K_{2}(s, t) \frac{\partial \varphi_{i}}{\partial t}\right)- \\
& -1 / 2 m^{2} K_{3}(s, t) \varphi_{i}(s, t)+K_{4}(s, t) v(s, t) \varphi_{i}(s, t) \\
& =\varepsilon_{i} K_{4}(s, t) \varphi_{i}(s, t)
\end{aligned}
$$

Similary we get for the Poisson equation (4)

$$
\begin{aligned}
& =\frac{1}{2} \frac{\partial}{\partial s}\left(k_{1}(s, t) \underset{\partial V^{c}}{---}\right)-\frac{1}{\partial s} \frac{\partial}{2} \frac{\partial t}{\partial t}\left(k_{2}(s, t) \frac{\partial V^{c}}{\partial t}\right) \\
& =-4 \pi k_{4}(s, t) \rho(s, t)
\end{aligned}
$$

with

$$
\begin{aligned}
& K_{1}(s, t)=K_{2}(s, t)=R / 2 \sinh (s) \sin (t) \\
& K_{3}(s, t)=R / 2\left(\sinh ^{2}(s)+\sin ^{2}(t)\right) /(\sinh (s) \sin (t)) \\
& K_{4}(s, t)=R^{3} / 2\left(\sinh ^{2}(s)+\sin ^{2}(t)\right) \sinh (s) \sin (t)
\end{aligned}
$$

\section{THE FINITE ELEMENT METHOD $1-3$}

The FEM orginally was developed in engineering science in order to calculate static and dynamic stresses of complicated constructions. In order to apply the FEM to the solution of the Hartree-Fock-Slater equations, one best starts from the equivalent variational principle 
278

$$
\begin{aligned}
I_{1}=\iint t & -\frac{1}{4} k_{1}\left(\begin{array}{l}
\partial \varphi_{i}^{2} \\
-\frac{1}{\partial}
\end{array}\right)-\frac{1}{4} k_{2}\left(\begin{array}{l}
\partial \varphi_{i} \\
-\frac{1}{\partial t}
\end{array}\right)- \\
& \left.-\frac{1}{4} m^{2} k_{3}\left(\varphi_{i}\right)^{2}-\frac{1}{2}\left(V-\varepsilon_{i}\right) k_{4}\left(\omega_{i}\right)^{2}\right\} d s d t
\end{aligned}
$$

for the Schrödinger type equation (9) and

$$
\begin{aligned}
I_{2}=\iint\{ & -\frac{1}{2} K_{1}\left(\begin{array}{c}
\partial V^{c}{ }^{2} \\
---
\end{array}\right)-\frac{1}{\partial s} K_{2}\left(\begin{array}{c}
\partial V^{c} \\
---
\end{array}\right) \\
& \left.+4 \pi K_{4}(s, t) \rho(s, t) V^{c}\right\} d s d t
\end{aligned}
$$

for the Poisson equation (10).

We subdivide the two dimensional space by the use of triangles. On each element a number of points, the nodal points, are chosen. If $u^{e}(s, t)$ is the approximation of the unknown function on the element with the number e, this function is written as a linear combination of the nodal values

$$
u^{e}(s, t)=\sum_{i=1}^{p} u_{i}^{e} N_{i}^{e}(s, t)
$$

were $u_{i}^{e}$ is the $i-t h$ nodal value of the e-th element. The function $N_{j}^{e}(s, t)$ is the $i-t h$ shape function. The sum runs over all p nodal values of the element.

Because of the subdivision of the region into $\mathrm{N}$ elements the functionals 1 equ. (12) and $I_{2}$ equ. (13) can be written as a sum of element integrals

$$
I_{1}=\sum_{e=1}^{N} I_{1}^{e} \quad \text { and } \quad I_{2}=\sum_{e=1}^{N} I_{2}^{e}
$$

The substitution of the trial function (14) into the element integrals (15) leads to

$$
I_{1}^{e}=\left(\underline{u}^{e}\right)^{t} \cdot \underline{H}^{e} \cdot \underline{u}^{e}-\left(\underline{u}^{e}\right)^{t} \cdot \underline{s}^{e} \cdot \underline{u}^{e}
$$

with the matrix elements

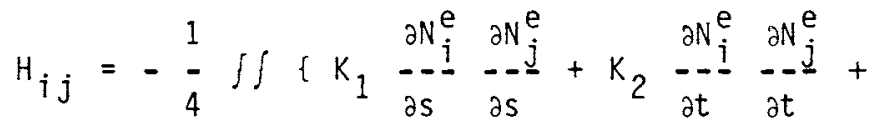

$$
\begin{aligned}
& \left.+\left(k_{3} m^{2}+2 k_{4} v\right) N_{j}^{e} N_{j}^{e}\right\} d s d t
\end{aligned}
$$

and 


$$
S_{i j}=\iint \frac{1}{2} K_{4} N_{j}^{e} N_{j}^{e} d s d t
$$

and for the Poisson equation similary

$$
I_{2}^{e}=\left(\underline{u}^{e}\right)^{t} \cdot \underline{D}^{e} \cdot \underline{u}^{e}-\left(\underline{u}^{e}\right)^{t} \cdot \underline{d}^{e}
$$

with the matrix elements

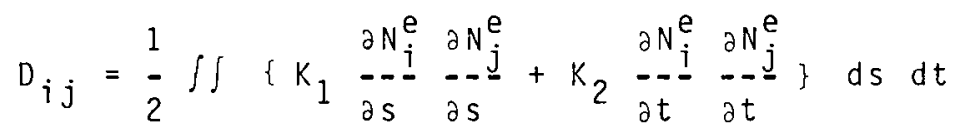

and the vector elements

$$
d_{i}=4 \pi \iint k_{4} \rho(s, t) N_{i}^{e} d s d t \quad .
$$

The vector $\underline{u}^{e}$ is the element nodal vector of the e-th element.

Adding the contributions of all elements leads to the expressions:

$$
I_{1}=\underline{u}^{t} \cdot \underline{H} \cdot \underline{u}-\underline{u}^{t} \cdot \underline{s} \cdot \underline{u}
$$

and

$$
I_{2}=\underline{u}^{t} \cdot \underline{D} \cdot \underline{u}-\underline{u}^{t} \cdot \underline{d}
$$

where $\underline{u}^{t}$ denotes the global nodal vector. Minimizing I and I with respect to the nodal values leads to the matrix eqgenvalue problem

$$
\underline{H} \cdot \underline{u}=\varepsilon \cdot \underline{S} \cdot \underline{u}
$$

for the schrödinger type equation and to the matrix equation

$$
\underline{D} \cdot \underline{u}=\underline{d}
$$

for the poisson equation.

The calculation of the matrix elements $(17,18,20,21)$ is done $_{6}$ numerically with a conical product Gauss integration rule ${ }^{6}$.

To account for the asymptotic decrease of the potential $V^{C}$ we introduce a function $g(s, t)$

$$
v^{c}=g(s, t) \cdot f^{v}(s, t)
$$

and solve for the function $f^{v}(s, t)$ by the FEM. 


\section{RESULTS}

For the calculations the points were distributed equidistant in both coordinates for simplicity. Such an equidistant point distribution is not at all optimal and it will be shown that point distributions which are physically more adequate will increase the accuracy.

In Tab. I we compare the convergence of the results with increasing grid size for both 5 -th and for 6-th order polynomials for the system $\mathrm{N}_{2}$. These results are expected to be accurate within $10^{-8}$ a.u.

Table I :

$\mathrm{N}_{2}$, interquclear distance $\mathrm{R}=2.07 \mathrm{a} . \mathrm{u}$.

Laksonen this work, 5-th order polynomials

$\begin{array}{lrrr}\text { Points } & 5989 & 3136 & 4356 \\ E_{(} & -108.346622 & -108.3466076 & -108.3466090 \\ (1 \sigma g) & -13.981070 & -13.98106828 & -13.98106840 \\ (1 \sigma u) & -13.979661 & -13.97965838 & -13.97965850 \\ (2 \sigma g) & -1.007215 & -1.00721472 & -1.00721471 \\ (2 \sigma u) & -0.460725 & -0.46072505 & -0.46072505 \\ (1 \pi u) & -0.404235 & -0.40423462 & -0.40423462 \\ (3 \sigma g) & -0.350058 & 0.35005852 & -0.35005852\end{array}$

this work, 6-th order polynomials

$\begin{array}{lrrr}\text { Points } & 2401 & 3025 & 4489 \\ E_{(} & -108.3466090 & -108.34660925 & -108.34660934 \\ (1 \sigma g) & -13.9810686 & -13.98106844 & -13.98106844 \\ (1 \sigma u) & -13.9796587 & -13.97965854 & -13.97965854 \\ (2 \sigma g) & -1.00721474 & -1.00721471 & -1.00721471 \\ (2 \sigma g) & -0.46072506 & -0.46072505 & -0.46072505 \\ (1 \pi u) & -0.40423461 & -0.40423462 & -0.40423462 \\ (3 \sigma g) & -0.35005852 & 0.35005852 & -0.35005852\end{array}$

Total energy and energy eigenvalues of the system $\mathrm{N}_{2}$ for different grid sizes and different order of the polynomials on the elements. For the largest grids the last figure is uncertain. All values are given in a.u. .

In Tab. I I a step towards an optimized point distribution is documented. In this table we compare the results for the system $N_{2}$ for a $31 * 31$ points grid of 6 -th order. In mesh $A$ we use an equidistant distribution of the points whereas in mesh B the s-coordinates (Equ. 7) of the vertices of the triangles are distributed logarithmically improving the accuracy by about a factor of 15 . 
Table I1:

$\mathrm{N}_{2}$, internuclear distance $\mathrm{R}=2.07 \mathrm{a} . \mathrm{u}$. 6 th order polynomials

$\begin{array}{ccc} & \text { Mesh } & \text { Mesh B } \\ \text { Points } & 961 & 961 \\ { }^{E}(1 \sigma) & -108.34645 & -108.34659 \\ (1 \sigma g) & -13.98104 & -13.981066 \\ \left(1 \sigma^{u}\right) & -13.97963 & -13.979656 \\ \left(2 \sigma^{g}\right) & -1.0072143 & -1.0072150 \\ \left(1 \pi_{u}^{u}\right) & -0.4607255 & -0.4607253 \\ \left(3 \sigma_{g}^{u}\right) & -0.4042346 & -0.4042347 \\ & -0.3500577 & 0.3500587\end{array}$

Total energy and energy eigenvalues of the system $\mathrm{N}_{2}$ for two different point distributions? Mesh A refers to a equidistant grid with all elements of equal size whereas mesh $B$ refers to a $10-$ garithmically point distribution in the s-coordinate. All values are given in a.u.

The results presented are by 2 orders of magnitude more accurate then the results achieved by Laaksonen et al.4 with the finite difference method, although we used a much smaller number of points.

A Hartree-Fock approach to diatomic molecules will be one of our next goals. The first attempt towards an optimized element distribution shows a very nice increase in accuracy. With better adapted element distributions one may tackle the 3-dimensional problems within reasonable computer times.

Acknowledgement

This work was partially supported by the Deutsche Forschungsgemeinschaft (DFG) and the Gesellschaft fuir Schwerionenforschung Darmstadt (GSI).

\section{References}

1. G. Strang, G. Fix, An Analysis of the Finite Element

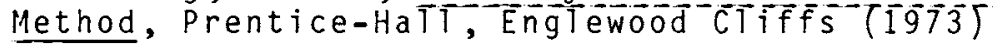

2. D.H. Norrie, G. de Vries, An Introduction to Finite Element Analysis, Academic Press, New York (1978) 
3. H.R. Schwarz, Methode der finiten Elemente, Teubner, Stuttgart (19 $\overline{8} 0)$

4. L. Laaksonen, D. Sundholm and P. Pyykkö, Comp. Phys. Rep. 4 , 313 (1986)

5. A.D. Becke, J. Chem. Phys. $\underline{76}, 6037$ (1982)

6. A.H. Stroud, Approximate calculation of multiple integrals, Prentice-HaTT, Englewood CTiffs (I971) 